\title{
ESTRATEGIAS DE INSERCIÓN INTERNACIONAL DEL MERCOSUR Y LA ASEAN
}

\section{STRATEGIES FOR THE INTERNATIONAL INSERTION OF MERCOSUR AND ASEAN}

\author{
NATALIA DE MARÍA* \\ MARÍA EUGENIA PEREIRA**
}

Recibido 29 de julio del 2016 Aceptado 2 de septiembre del 2016 

Revista PuCe. IsSn 1390-7719. Núm.103. 3 de mayo de 2016-

3 De noviembre de 2016. Natalia De María,

María Eugenia Pereira.PP. 273-297

\section{ESTRATEGIAS DE INSERCIÓN INTERNACIONAL DEL MERCOSUR Y LA ASEAN}

\section{STRATEGIES FOR THE INTERNATIONAL INSERTION OF MERCOSUR AND ASEAN}

\section{NATALIA DE MARÍA, MARÍA EUGENIA PEREIRA}

PALABRAS CLAVE: Integración, inserción internacional, acuerdos, MERCOSUR, ASEAN

KEY WORDS: Integration, international insertion, agreement, MERCOSUR, ASEAN

\section{RESUMEN}

El artículo analiza las acciones en materia de inserción internacional que llevan adelante dos procesos de integración líderes en sus respectivas regiones como lo son el MERCOSUR la ASEAN. Para ello se estudiarán los acuerdos que concretaron cada uno de ellos y la agenda externa que plantean los bloques.

\section{ABSTRACT}

This article analyzes actions for international insertion that two integration processes pursue regarding the leaders in their respective regions represented by MERCOSUR and ASEAN. Agreements will be studied, achieved by each one, as well as the external agenda proposed by the blocks involved. 


\section{INTRODUCCIÓN}

Tanto el Mercado Común del Sur (MERCOSUR) como la Asociación de Naciones del Sudeste Asiático (ASEAN), son dos procesos de referencia en sus respectivas regiones. Sin embargo, sus orígenes y evolución son distintos. Mientras que el MERCOSUR nace como un acuerdo que se compromete a alcanzar una integración profunda, en donde la inserción internacional de sus miembros queda subordinada al bloque, la ASEAN no presenta en sus inicios un interés marcado en coordinar sus acciones de inserción internacional y deja este tema libre a la voluntad de sus Estados Parte.

A través del análisis de los textos fundacionales de ambos bloques, de sus instrumentos de integración, así como de los acuerdos firmados por cada uno, se intentará identificar los objetivos fundacionales en materia de inserción, así como la evolución de cada uno en cuanto a sus intereses y caminos para concretar el alcance de los objetivos establecidos. Asimismo, se realizará una comparación de las distintas estrategias de inserción internacional de ambos procesos y se analizará la situación actual de cada uno en cuanto al cumplimiento de la misma y los logros alcanzados.

En momentos de cambios tanto a nivel global en donde las instituciones multilaterales se ven seriamente afecta- das, así como también en las regiones que incluyen al MERCOSUR y la ASEAN, merece la pena comparar las estrategias que llevaron adelante ambos bloques, así como cuáles fueron las formas de inserción internacional elegidas por cada uno y qué tan efectivas fueron las mismas para su inserción en un mundo globalizado y cambiante. Estas cuestiones cobran relevancia para explicar el desempeño de estos procesos de integración y determinar en qué medida lograron alcanzar sus objetivos con las estrategias utilizadas.

\section{La inserción internacional del MERCOSUR}

El 26 de marzo de 1991 se firma el Tratado de Asunción, que conformó el MERCOSUR. Este hecho marca un hito en la historia sudamericana, no solo por lo que este acuerdo representa a nivel económico comercial, sino también por el peso político que conlleva al lograr un acercamiento entre Argentina y Brasil, dos de las principales economías de la región. Es indiscutible que el nacimiento del mismo tiene connotaciones geopolíticas, además de económicas. Sin embargo, el foco del presente artículo se centra en las acciones que los países miembros realizan con el fin de concretar una inserción internacional beneficiosa. 
Revista PUCE. ISSN 1390-7719. Núm.103. 3 de mayo de 2016 -

3 De noviembre de 2016. Natalia De María,

María Eugenia Pereira.PP. 273-297

Este acuerdo va a marcar un precedente en la política comercial de los cuatro Estados originarios del bloque: Argentina, Brasil, Paraguay y Uruguay. El artículo primero del Tratado de Asunción expresa que el objetivo fundamental será la conformación de un mercado común, lo que determina que se libere la circulación de factores productivos y el establecimiento de un arancel externo común. Sobre este tema específicamente, el documento menciona:

...establecimiento de un arancel externo común y la adopción de una política comercial común con relación a terceros Estados o agrupaciones de Estados y la coordinación de posiciones en foros económico-comerciales-regionales e internacionales. ${ }^{\text {? }}$

La inserción internacional comercial de los países miembros va a estar unida desde el momento en que el MERCOSUR nace, tal como lo menciona el artículo primero. Desde entonces, los países se comprometen a coordinar posiciones en lo que se refiere a asuntos económicos y regionales, además de crear un arancel externo común, herramienta que los obliga a coordinar sus

\footnotetext{
${ }^{1}$ MERCOSUR (1991) Tratado de Asunción.
}

políticas comerciales lo que genera una política que sea única y que represente al bloque en su conjunto. Sin embargo, el Tratado de Asunción no hace referencia a la forma de cómo alcanzar los objetivos planteados, por lo que necesariamente se necesitará de otros documentos que resuman las acciones a llevar a cabo (Bizzozero \& Vera, 1995).

El compromiso de formar un proceso de integración de corte profundo es un hecho interesante por lo que ello implica. En la década del noventa, la mayor parte de los acuerdos notificados ante la Organización Mundial de Comercio (OMC) eran zonas de libre comercio por la relativa facilidad para su implementación entre los Estados interesados, además de la independencia que este tipo de instrumento genera en la política comercial de los miembros (Bartesaghi, 2015a). A pesar de ello, el MERCOSUR surge con el ambicioso objetivo de transformarse en un único territorio aduanero. A los países miembros esto le resultaba conveniente por diversas razones: en primer término, Brasil y Argentina veían al bloque como una forma de acercamiento entre sus principales socios comerciales, además de traer estabilidad a la región y además era para Brasil la posibilidad de confirmar su situación como potencia regional. Luego, para Uruguay y Paraguay quedarse fuera de un acuerdo con dos de sus so- 
cios principales no era una opción, además de que la promesa de negociar un acuerdo en bloque, aumenta el poder negociador.

Durante los primeros nueve años, el bloque se orientó hacia el objetivo de alcanzar las metas planteadas en su tratado fundacional y en protocolos firmados posteriormente como por ejemplo el de Ouro Preto (1994), a partir del cual se comienza a conformar la unión aduanera. Es en este período donde el MERCOSUR muestra un mayor avance, puesto que elimina las barreras internas al comercio, aumenta el intercambio intrazona de bienes y consolida su arancel externo común (Peña, 1996). Se debe mencionar también que desde que se firma el Protocolo de Ouro Preto, el MERCOSUR adquiere personería jurídica lo cual hace que el bloque pueda firmar acuerdos con terceros y converge las distintas políticas comerciales de los Estados miembros en una sola (anteriormente a este hecho, en caso de firmarse o negociarse un acuerdo debía hacerse bajo el modelo 4+1 como se planteó en 1991 con Estados Unidos). Es en este período que se firma el primer acuerdo del bloque con Chile, que se enmarcó en el ámbito de la Asociación Latinoamericana de Integración (ALADI), organismo al cual pertenecen los miembros del MERCOSUR y otras nueve economías latinoamericanas y que funciona como acuerdo marco para propulsar e incentivar la integración regional, por el acuerdo ACE N³5.

En el año 2000, el bloque lanza la denominada "Agenda de Relanzamiento del MERCOSUR" debido a la necesidad de impulsar el proceso hacia la conformación del mercado común, tras sucesos como la devaluación de Brasil en el año 1999. Es en ese momento donde se incorporan diferentes decisiones entre las que se encuentra la N³2/00 (Fernández Reyes, 2014), que terminará de delimitar la agenda externa del bloque. El artículo 1 de la misma expresa:

Reafirmar el compromiso de los Estados Partes del MERCOSUR de negociar en forma conjunta acuerdos de naturaleza comercial con terceros países o agrupaciones de países extrazona en los cuales se otorguen preferencias arancelarias. $^{2}$

Más allá de los cuestionamientos que rodean a la decisión (Fernández Reyes, 2015), entre los que se encuentra el hecho que no está incorporada por ninguno de los Estados miembros, la misma condiciona hasta la fecha la inserción internacional del bloque. Es también uno de los cuestionamientos más grandes

${ }^{2}$ MERCOSUR/CMC/DEC N ${ }^{\circ} 32 / 00$. 
Revista PUCE, ISSN 1390-7719. Núm.103. 3 de MAYO de 2016 -

3 De noviembre de 2016. Natalia De María,

María Eugenia Pereira.PP. 273-297

que se le hace al MERCOSUR por parte de sus propios miembros. Uruguay, por ejemplo, es uno de los países que solicitó en varias oportunidades que se flexibilice esta medida y se permita la negociación con otros países (bilateral o a distintas velocidades) por parte de los Estados miembros. Se considera que el bloque no firma acuerdos con grandes mercados (no se cuenta con economías desarrolladas, ni con los principales actores asiáticos, salvo India), por el hecho de que no se permite que los países miembros lo hagan por su cuenta, esto es aislar al bloque en un mundo cada vez más interconectado.

Diferentes motivos hicieron que el MERCOSUR se aleje de sus objetivos originarios y comience a darle peso a otros ámbitos, como el fortalecimiento de aspectos sociales y culturales. Durante los últimos años, las políticas del bloque giraron hacia otros temas, entre los cuales la agenda externa no es una prioridad. Un análisis de las decisiones adoptadas por el Consejo Mercado Común durante los años 2013 a 2015 muestra que solamente el $15 \%$ de sus decisiones tuvo relación con la agenda externa del bloque y salvo por la adhesión de Bolivia, los impactos de la misma en términos comerciales son bajos, lo que demuestra el estancamiento del proceso en cuanto a su agenda externa (Bartesaghi, 2015b).

\section{Acuerdos firmados por el MERCOSUR}

Porque el MERCOSUR se encuentra en el marco de la ALADI (ACP.CE N ${ }^{\circ} 18$ ), implica la intención de los miembros de continuar su integración con la región, se divide el análisis entre los acuerdos firmados intrazona (dentro de la ALADI) y extrazona. El MERCOSUR firmó siete acuerdos en el marco de la ALADI, además de tres para formar zonas de libre comercio (Israel, Egipto y Palestina) y dos de preferencias fijas (SACU e India). Los primeros acuerdos que alcanzó el MERCOSUR son con los países de la región (con los que, como se mencionó anteriormente) ya tenía un acercamiento a través de la ALADI, tal como se muestra en el cuadro que se presenta a continuación.

\section{CUADRO 1}

ACUERDOS DEL MERCOSUR EN EL MARCO DE LA ALADI

\begin{tabular}{|c|c|c|}
\hline AAP.CE & País & Año \\
\hline$N^{\circ} 35$ & Mercosur - Chile & 1996 \\
\hline$N^{0} 36$ & Mercosur - Bolivia & 1997 \\
\hline$N^{\circ} 54$ & Mercosur - México & 2003 \\
\hline$N^{\circ} 55$ & Mercosur - México & 2004 \\
\hline$N^{0} 58$ & Mercosur - Perú & 2005 \\
\hline & Mercosur - Colombia, & \\
\hline$N^{\circ} 59$ & Ecuador y Venezuela & 2004 \\
\hline$N^{0} 62$ & Mercosur - Cuba & 2008 \\
\hline
\end{tabular}

Fuente: elaboración propia a base de datos extraídos de la ALADI.

El primer acuerdo que firma el MERCOSUR es con Chile. Este hecho no sorprende sí se toma en cuenta que en los inicios del bloque, el país andino fue invitado a participar en el acuerdo y rechazó la oferta por considerar que 
los aranceles que proponía el naciente proceso eran superiores a los que ya aplicaba ese país. Lo cierto es que Chile desde su alejamiento de la Comunidad Andina (CAN), comienza a practicar una estrategia que consistía en la rebaja de sus aranceles de forma unilateral mientras que cerraba acuerdos bilaterales con diferentes países. (Bartesaghi, 2010a). El acuerdo que se formalizó ante la ALADI como AAP.CE N³5 establece una zona de libre comercio entre los miembros que se pretendía establecer para el año 2015. Uruguay y Chile cumplen con ese objetivo con excepciones muy puntuales. Desde la firma del mismo, se concretaron 56 protocolos adicionales en diversos temas relativos al comercio de bienes y de servicios.
En cuanto a los acuerdos firmados con terceros países (extra ALADI), se debe destacar el que se cuenta con India. El mismo tiene la característica de ser limitado en cuanto a su alcance, no solo por la escasa cantidad de líneas arancelarias que se incluyen, sino también porque no se prevé un cronograma de liberalización (Bartesaghi, 2010b). Sin embargo, es un acuerdo de gran relevancia para el bloque si se considera que es el único con un país asiático que es, el segundo en términos de población y con una economía que crece por encima del promedio mundial, superando incluso a China en los últimos años. Una ampliación de los términos negociados sería positiva para el bloque.

\section{CUADRO 2}

ACUERDO CON PAÍSES EXTRAZONA ALADI

\begin{tabular}{|c|c|c|}
\hline Tipo de Acuerdo & Año & Comentarios \\
\hline \multicolumn{3}{|c|}{ Acuerdo para la creación de una ZLC/TLC } \\
\hline Israel & 2007 & Entrada en vigor en el 2011 \\
\hline Egipto & 2010 & Pendiente la ratificación de Argentina y Paraguay \\
\hline Palestina & 2011 & Pendiente de ratificación de los Estados Parte \\
\hline \multicolumn{3}{|c|}{ Acuerdo preferencial de comercio } \\
\hline India & 2004 & Entrada en vigor en el 2009 \\
\hline SACU & 2004 & Entrada en vigor en el 2015 \\
\hline
\end{tabular}

Fuente: elaboración propia a base de datos extraídos del MERCOSUR.

Al observar los acuerdos que firmó el MERCOSUR, se puede mencionar que salvo algunos casos puntales, no los posee con sus principales socios comerciales. Dentro de los destinos de exportación más importantes de los paí- ses miembros durante el año 2015 (sin tomar en cuenta el comercio intrazona) solamente se tiene un acuerdo con India (el $4 \%$ de las exportaciones tienen como destino este país), el que, como se mencionó previamente, es un acuerdo muy 
ReVista PUCE, ISSN 1390-7719. Núm.103. 3 de mayo de 2016-

3 De noviembre de 2016. Natalia De María,

María Eugenia Pereira.PP. 273-297

limitado en cuanto a líneas arancelarias negociadas y otro con Chile. El resto de los países que componen el top 10 de las exportaciones son China, Estados Unidos y los países miembros de la Unión Europea. En el caso de las importaciones, el único Estado con el que se tiene un acuerdo que figura entre los principales proveedores del MERCOSUR, es México. Nuevamente, China, Estados Unidos y países europeos son los que completan el "ranking".

CUADRO 3

DESTINO Y ORIGEN DE LAS EXPORTACIONES DEL MERCOSUR DURANTE EL 2015

\begin{tabular}{|c|c|}
\hline \multicolumn{2}{|c|}{ Destinos de las exportaciones } \\
\hline País & Participación \\
\hline China & $16 \%$ \\
\hline Estados Unidos & $15 \%$ \\
\hline Brasil & $5 \%$ \\
\hline Argentina & $5 \%$ \\
\hline India & $4 \%$ \\
\hline Países Bajos & $4 \%$ \\
\hline Alemania & $2 \%$ \\
\hline Chile & $2 \%$ \\
\hline Japón & $2 \%$ \\
\hline España & $2 \%$ \\
\hline
\end{tabular}

\begin{tabular}{|c|c|}
\hline \multicolumn{2}{|c|}{ Origen de las importaciones } \\
\hline País & Participación \\
\hline China & $19 \%$ \\
\hline Estados Unidos & $16 \%$ \\
\hline Brasil & $7 \%$ \\
\hline Alemania & $5 \%$ \\
\hline Argentina & $5 \%$ \\
\hline México & $3 \%$ \\
\hline Corea & $3 \%$ \\
\hline Italia & $2 \%$ \\
\hline Japón & $2 \%$ \\
\hline Francia & $2 \%$ \\
\hline
\end{tabular}

Fuente: elaboración propia a base de datos extraídos de Trade Map.

La mayor parte de los acuerdos que firmó el MERCOSUR no representan un gran porcentaje en el comercio total de productos. Si se observan las exportaciones del bloque durante 2015, poco más del $12 \%$ son bienes que se colocan en algún mercado con el que se tiene un acuerdo (este porcentaje representa menos de lo que el bloque exporta hacia China o Estados Unidos). Si se desagrega por regiones, las exportaciones al resto de los países de la ALADI con los que se posee un acuerdo son más significativas que las ventas a los mercados extrazo- na con los que también se cuenta con un tratado. Chile y México se muestran como dos socios importantes, ubicándose en los primeros puestos como mercado de destino de las exportaciones. Además, se debe destacar el rol que juega India, el cual se presenta como el quinto destino de las exportaciones del bloque, a pesar de que el acuerdo es de alcance limitado. En cuanto a las importaciones, la relevancia de los socios comerciales es aún más limitada que en las exportaciones, porque representan en el año 2015 poco más de un $10 \%$. Nuevamente se ve 
una mayor importancia de las importaciones provenientes de los países de la
ALADI, las cuales representan un 7\% del total de las adquisiciones.

\section{CUADRO 4}

COMERCIO EXTERIOR CON LOS PAÍSES CON LOS QUE EL MERCOSUR TIENE ACUERDO

\begin{tabular}{|c|c|c|c|c|c|}
\hline & Exportaciones & & & Importaciones & \\
\hline Pais & Participación & Posicion & Pais & Participación & Posicion \\
\hline India & $4,13 \%$ & 5 & India & $1,98 \%$ & 12 \\
\hline Egipto & $1,09 \%$ & 24 & Egipto & $0,04 \%$ & 71 \\
\hline Israel & $0,28 \%$ & 51 & Israel & $0,38 \%$ & 41 \\
\hline Palestina & $0,02 \%$ & 114 & Palestina & $0,00 \%$ & 162 \\
\hline SACU & $0,69 \% *$ & $155^{* *}$ & SACU & $0,30 \% *$ & $147^{* *}$ \\
\hline Sub total & $5,52 \%$ & & Sub total & $2,40 \%$ & \\
\hline Chile & $2,35 \%$ & 8 & Chile & $1,72 \%$ & 14 \\
\hline México & $1,61 \%$ & 11 & México & $2,79 \%$ & 6 \\
\hline Perú & $0,95 \%$ & 29 & Perú & $0,57 \%$ & 32 \\
\hline Colombia & $0,94 \%$ & 30 & Colombia & $0,91 \%$ & 23 \\
\hline Bolivia & $0,73 \%$ & 37 & Bolivia & $1,47 \%$ & 15 \\
\hline Ecuador & $0,32 \%$ & 48 & Ecuador & $0,27 \%$ & 52 \\
\hline Cuba & $0,27 \%$ & 53 & Cuba & $0,03 \%$ & 85 \\
\hline Sub total & $7,16 \%$ & & Sub total & $7,76 \%$ & \\
\hline Total & $12,68 \%$ & & Total & $10,16 \%$ & \\
\hline
\end{tabular}

Fuente: elaboración propia a base de datos extraídos de Trade Map.

Asimismo, el MERCOSUR firmó una serie de acuerdos marco y memorando de entendimiento, los cuales aún deben ser ratificados por los países miembros. Si se observa el cuadro $\mathrm{N}^{0} 4$ se puede apreciar que ninguno de ellos representa un mercado de gran importancia para el bloque.

\section{CUADRO 5}

MEMORANDO DE ENTENDIMIENTO Y ACUERDOS MARCO DEL MERCOSUR

\begin{tabular}{|c|c|c|}
\hline Tipo de Acuerdo & Año & Comentarios \\
\hline \multicolumn{3}{|l|}{ Memorándum de entendimientos } \\
\hline Guyana & 1999 & No entró en vigor \\
\hline Trinidad y Tobago & 1999 & No entró en vigor \\
\hline Singapur & 2000 & Incluye Inversiones. No entró en vigor \\
\hline Corea & 2009 & $\begin{array}{c}\text { En vigor. Creación de un grupo consultivo conjunto para la promoción del comercio y las } \\
\text { inversiones entre el Mercosur y Corea }\end{array}$ \\
\hline Libano & 2014 & Pendiente la ratificación de Brasil, Paraguay, Uruguay y Venezuela \\
\hline \multicolumn{3}{|l|}{ Acuerdos Marco } \\
\hline India & 2003 & Pendiente la ratificación de India \\
\hline Egipto & 2004 & Falta la aprobación de Egipto \\
\hline Marruecos & 2004 & Entró en vigor en el año 2010 \\
\hline Consejo de Cooperación del Golfo & 2005 & Pendiente de ratificación por el CCG \\
\hline Israel & 2005 & Pendiente de ratificación. \\
\hline Pakistán & 2006 & Pendiente la ratificación de Paraguay y Pakistán \\
\hline Jordania & 2008 & Entró en vigor en el año 2011 \\
\hline Turquía & 2008 & Pendiente la ratificación de Paraguay y Turquía \\
\hline Siria & 2010 & Pendiente la ratificación de Paraguay, Uruguay y Siria \\
\hline Palestina & 2010 & Pendiente la ratificación de Paraguay, Uruguay y Palestina \\
\hline Túnez & 2014 & Pendiente la ratificación de Brasil, Paraguay, Uruguay y Túnez \\
\hline Surinam & 2015 & Pendiente la ratificación de todos los Estados Parte \\
\hline
\end{tabular}

Fuente: elaboración propia a base de datos extraídos del MERCOSUR. 
Revista PUCE. ISSN 1390-7719. Núm.103. 3 de mayo de 2016 -

3 De noviembre de 2016. Natalia De María,

María Eugenia Pereira.PP. 273-297

Al dejar de lado los acuerdos que, firmados con otros miembros de la ALADI, la agenda que el bloque lleva adelante con el fin de acercar al MERCOSUR a las grandes economías y bloques a nivel mundial, es escasa en cuanto al cierre de tratados. La posibilidad de revertir esta situación se encuentra en la concreción del acuerdo con la Unión Europea, el que lleva negociándose hace 17 años y de las que solo participan los miembros originarios, queda fuera Venezuela. Habrá que ver el impacto que tendrá el alejamiento de Reino Unido de este bloque y si esto significará un obstáculo más en las negociaciones que se llevan adelante con el MERCOSUR. A este aspecto se le debe sumar que la Unión Europea lleva adelante otras negociaciones de forma paralela, como por ejemplo el Transatlantic Trade and Investment Partnership (TTIP) con Estados Unidos.

Se puede concluir, que son fácilmente identificables dos etapas en la firma de acuerdos que lleva adelante el bloque. En la primera se identifica claramente la firma de tratados con los países miembros de la ALADI, que son los que mayor importancia representan si se toman en cuenta sus participaciones en el total del comercio. En esta etapa entraría también el acuerdo firmado con India que a pesar de la cobertura parcial, representa un acercamiento a una eco- nomía emergente importante y el único acuerdo con Asia. Además, en la segunda etapa se firman acuerdos que tienen un interés más político que comercial (Israel, Palestina, Egipto, además de Cuba que si bien pertenece a la ALADI es un acuerdo que refleja intereses políticos y tiene escasa significancia económica), hechos que coinciden con la politización que tuvo el bloque en sus diferentes áreas de acción.

De aquí en más, el bloque podría abrir un nuevo capítulo en su historia. Los cambios en el gobierno de Argentina, que se suma a la crisis institucional de Brasil que colocó en el poder (al menos de forma temporal), a un gobierno con ideas opuestas en cuanto a inserción internacional seguida por Dilma Rousseff, más la continua insistencia de Uruguay de abrir nuevos mercados, podría materializarse en un cambio de rumbo de la política comercial conjunta. Cada vez son más las voces que claman por una flexibilización en el MERCOSUR, planteándose la posibilidad de habilitar la opción que se negocien acuerdos de forma bilateral, o al menos, en distintas velocidades, lo que significaría sumar una excepción más a una unión aduanera imperfecta, en el caso que no quiera revisarse el instrumento de integración elegido hace años atrás.

El MERCOSUR atraviesa un momento crítico en su historia, donde los 
cambios en los gobiernos de los dos socios mayores del bloque podrían dar cabida a un escenario en el que el mismo se reformulase. En un contexto donde el ámbito multilateral no arroja los resultados que se esperan y donde las nuevas tendencias en el comercio internacional hacen que cada vez sea más necesaria, la incorporación de nuevas normas que regulen los intercambios de bienes y servicios, es importante que los procesos de integración contribuyan con normas que suplan los vacíos que dejan los sistemas jurídicos nacionales. Lo cierto es que el MERCOSUR se encuentra atrapado en un bloque que pasó los últimos años encerrándose en sí mismo, mientras no muy lejos, países vecinos cierran acuerdos innovadores y con temas actuales, como es el caso de la Alianza del Pacífico y su Protocolo Comercial, el que entró en vigor recientemente. Tres de los miembros de dicho proceso de integración además participan de las meganegociaciones comerciales como el Trans-Pacific Partnership (TPP). EI MERCOSUR, entonces, debe resolver el rumbo que quiere tomar en los próximos años, en donde deberá decidir qué intereses primarán: los políticos o los comerciales.

\section{Inserción internacional de la ASEAN}

La ASEAN es uno de los procesos de integración con mayor dinamismo en la actualidad. El bloque se crea el ocho de agosto de 1967 mediante la firma por parte de Indonesia, Malasia, Filipinas, Singapur y Tailandia de la Declaración de Bangkok, tratado fundacional del proceso. Posteriormente se unieron al bloque Brunéi en el año 1984, Vietnam en 1995, Laos y Myanmar en 1997 y finalmente Camboya en el año 1999, constituyéndose estos diez Estados en los actuales miembros de la ASEAN.

El motivo que llevó a estos países a integrarse fue fundamentalmente de carácter político y de seguridad; el impulso fue los enfrentamientos y tensiones existentes en la región del sudeste asiático e incluso algunos de ellos entre los propios miembros del bloque. Muchos de estos conflictos se fomentaron por la injerencia extranjera; hay que considerar que todos los países (salvo Tailandia) fueron colonias, además de la importancia geopolítica de su ubicación. De esta manera, como se expresa en la Declaración de Bangkok, deciden integrarse y conformar una Asociación para la Cooperación Regional con el objetivo de alcanzar la paz regional e impulsar su progreso y desarrollo, así como promover la prosperidad regional, afianzando los lazos de solidaridad y cooperación entre ellos.

Una de las características del bloque surge de su tratado fundacional, ya que el enfoque que tuvo en sus comien- 
Revista PUCE, ISSN 1390-7719. Núm.103. 3 de MAYO de 2016 -

3 De noviembre de 2016. Natalia De María,

María Eugenia Pereira.PP. 273-297

zos era exclusivamente político y de seguridad, dejo de lado el plano económico (Bartesaghi, 2014a), ámbito que por lo general suele tener una importancia significativa en todos los procesos de integración, los que generalmente suelen marcarse objetivos y compromisos para profundizar sus relaciones económicas y comerciales. En el caso de la ASEAN, si bien su Declaración hace referencia a que uno de los propósitos será fomentar el crecimiento económico, no se establecen compromisos en este sentido, sino como medio para potenciar la prosperidad y el equilibrio regional, en forma conjunta con los objetivos de desarrollo social y cultural. Queda claro entonces que, en su creación la ASEAN no tenía un cometido económico ni comercial y la inserción internacional del bloque no se encontraba entre sus metas en ese momento.

Respecto a la evolución del bloque, se identificaron por los expertos dos etapas, sí bien existen diferencias entre ellos, algunos consideran que esa nueva orientación se da a partir de la década del ochenta y otros que es en la del noventa como producto de la crisis financiera asiática. La segunda etapa sería la identificada con un claro perfil económico comercial (Bartesaghi, 2014b). Una vez que la paz se alcanza en la región, (lo cual se establece en una serie de declaraciones y acuerdos que sellan compro- misos en ese sentido) y con el fin de la Guerra Fría, los miembros de la ASEAN se enfocan en proyectar un crecimiento que apunta hacia el ámbito económico comercial y la inserción internacional.

El primer paso hacia esta nueva orientación se da en el año 1992 mediante la firma de un acuerdo para establecer un área de libre comercio, así como otros acuerdos sobre inversiones y servicios. Asimismo, en ese año como forma de potenciar esta nueva dinámica comercial del bloque es que se celebra la primera reunión del Foro Regional de la ASEAN, instancia abierta a la participación de aquellos países interesados y con el objetivo de promover el diálogo entre ellos. Además, comienzan a participar del foro Asia Pacific Economic Cooperation (APEC) y refuerzan la relación con la Unión Europea mediante el intercambio a través de reuniones denominadas Asia-Europe Meeting (ASEM).

Al celebrarse los 30 años del proceso de integración del sudeste asiático, los miembros deciden dar un paso más hacia la profundización de sus relaciones y suscriben un documento denominado ASEAN Vision 2020, que fortalece el giro del bloque hacia el ámbito económico comercial. En este documento se define lo siguiente:

We resolve to chart a new direction towards the year 2020 called, 
ASEAN 2020: Partnership in Dynamic Development which will forge closer economic integration within ASEAN. (ASEAN, 1997).

Otro paso importante hacia la nueva orientación se da en el marco de la ga Cumbre de Jefes de Estado y Gobierno en el año 2003, a través de la Declaración ASEAN Concord II, donde se define que se establecerá la Comunidad de la ASEAN, la cual se formará por tres pilares interrelacionados: político y seguridad, económico y socio-cultural. (ASEAN, 2003). A su vez, cada pilar conforma una Comunidad (ASEAN Political-Security Community, ASEAN Economic Community y ASEAN Socio-Cultural Community) que en su conjunto conforman la Comunidad de la ASEAN, la que en principio se propuso se instauraría en el año 2020, pero que posteriormente se adelanta dicha fecha hacia el año 2015. Es así que el 31 de diciembre de 2015 entró en funcionamiento la Comunidad de la ASEAN, lo cual demuestra el fuerte compromiso y voluntad política de los miembros en cumplir con lo que se asumió y deja en evidencia el pragmatismo del bloque en cuanto al alcance y puesta en marcha de los objetivos que se establecieron.

Si bien los tres pilares y sus respectivas comunidades son igualmente importantes, cabe hacer referencia al pilar económico, ya que entre sus objetivos está el de conformar un mercado único, lo cual implica la libre circulación de factores productivos. Asimismo, este pilar se enfoca en lograr que la región del sudeste asiático sea económicamente competitiva y dinámica, en promover un desarrollo económico equitativo e inclusivo, así como la integración de la ASEAN a la economía global, lograr una ASEAN resistente y enfocada hacia las personas. (ASEAN, 2015a). Es así que estos pilares reflejan claramente la estrategia de inserción del bloque en un mundo globalizado.

La meta de la conformación de la Comunidad se sustenta y fortalece mediante la Carta de la ASEAN que entró en vigor en el año 2008, documento que le otorga personería jurídica y un marco institucional al bloque, al ser un documento legalmente vinculante entre los Estados parte. La Carta, la cual fue registrada como corresponde en la Secretaría de Naciones Unidas, establece los objetivos y principios, así como también los derechos y obligaciones, entre otros temas relativos a la admisión de nuevos miembros y la creación de nuevos organismos para el funcionamiento del bloque. Pero la ASEAN no se quedó en el hecho de alcanzar su objetivo de formar la Comunidad y lo que ello implica, sino que reconocen que de aquí en más será una nueva etapa en cuan- 
Revista PUCE, ISSN 1390-7719. Núm.103. 3 de MAYO de 2016 -

3 De noviembre de 2016. Natalia De María,

María Eugenia Pereira.PP. 273-297

to a su correcto funcionamiento, por lo cual se establecen nuevos desafíos y compromisos para los próximos años, los cuales quedan plasmados en una serie de documentos y declaraciones que establecen nuevas metas hacia el año 2025.

Adicionalmente al compromiso y pragmatismo que caracterizan al bloque, se debe hacer referencia también a otro aspecto que forma parte del éxito del proceso y que es su flexibilidad. Los países que conforman la ASEAN poseen profundas diferencias y asimetrías en diversos aspectos, por lo cual la estrategia adoptada por el bloque permite que cada uno de los miembros incorpore los compromisos asumidos según sus propias posibilidades con el apoyo y cooperación del resto, promueve así el pragmatismo y sobre todo contempla las posibilidades de aquellos Estados parte con menor desarrollo.

Otro aspecto que se debe tener en cuenta al analizar el bloque del sudeste asiático es que se encuentra en proceso de conformar un mercado común, sin pasar por la etapa de una unión aduanera, salta de esta manera el orden lógico de etapas que contempla la teoría clásica de la integración. La ASEAN coordina su política comercial común a pesar que el modelo de integración que se eligió no implica la obligación de hacerlo. Si bien alcan- zan acuerdos en bloque, no poseen un arancel externo común, típico de una unión aduanera. De esta manera se promueve la flexibilidad ya que cada uno de los socios tiene la posibilidad de negociar libremente y cerrar acuerdos que dependen de su interés y capacidad negociadora. Esto deriva en que algunos de los miembros fueron muy activos en la firma de acuerdos con terceros (tal es el caso de Singapur); en cambio, otros países se enfocaron solamente en los acuerdos firmados en el marco de la ASEAN con terceros, acuerdos que se denominan ASEAN +1. Esto permitió potenciar la estrategia de inserción internacional de cada uno de los países con la de la ASEAN.

En el marco de los acuerdos de tipo ASEAN +1 , se encuentran vigentes acuerdos con China, Corea, Japón, India y uno en conjunto con Australia y Nueva Zelanda. Cada uno de estos incluye diferentes tipos de compromisos en materia de liberalización arancelaria, algunos incluso son muy pobres en cuanto a los compromisos alcanzados. Otros, como por ejemplo los acuerdos firmados con Australia y Nueva Zelanda, son más profundos y no se quedan solamente con la liberalización de tipo arancelaria, sino que abarcan otro tipo de temas referentes a las nuevas dinámicas del comercio internacional. 
CUADRO 6

ACUERDOS ASEAN +1

\begin{tabular}{|c|c|c|}
\hline ASEAN +1 & Alcance & Fecha de entrada en vigor \\
\hline Australia - Nueva Zelanda & Bienes y servicios & Enero 2010 \\
\hline China & Bienes $y$ servicios & Enero 2005 (Bienes), julio 2007 (Servicios) \\
\hline Corea & Bienes y servicios & Enero 2010 (Bienes), mayo 2009 (Servicios) \\
\hline India & Bienes y servicios & Enero 2010 (Bienes), julio 2015 (Servicios) \\
\hline Japón & Bienes & Diciembre 2008 \\
\hline
\end{tabular}

Fuente: Elaboración propia a base de OMC.

Si se analizan los 15 principales países de destino de las exportaciones de la ASEAN, así como los 15 principales países proveedores del bloque, surge que los países con los cuales tiene acuerdos de tipo ASEAN +1 son importantes socios comerciales, tanto en el caso de las exportaciones como de las importaciones. Se destaca China, prin- cipal socio comercial en ambos casos. Entre los 15 principales destinos de las exportaciones de la ASEAN cinco son con los que tiene acuerdos comerciales, los cuales representan el 33\% del total de sus exportaciones. Por su parte, entre los 15 principales países de origen de las importaciones realizadas por la ASEAN, se encuentran esos mismos cinco, que representan el 42\% del total de sus adquisiciones. Si bien Nueva Zelanda aún no se encuentra entre sus principales socios comerciales, cabe destacar que el comercio entre el bloque del sudeste asiático y este país crece significativamente en los últimos años gracias al acuerdo vigente.

\section{CUADRO 7}

PRINCIPALES SOCIOS COMERCIALES DE LA ASEAN

\begin{tabular}{|l|c|}
\hline \multicolumn{1}{|c|}{ Pais exportador } & $\begin{array}{c}\text { Participación en importaciones } \\
\text { totales de ASEAN (2015) }\end{array}$ \\
\hline China & $22 \%$ \\
\hline Japón & $9 \%$ \\
\hline Estados Unidos & $8 \%$ \\
\hline Singapur & $7 \%$ \\
\hline República de Corea & $7 \%$ \\
\hline Malasia & $6 \%$ \\
\hline Taipei Chino & $5 \%$ \\
\hline Tailandia & $5 \%$ \\
\hline Indonesia & $3 \%$ \\
\hline Alemania & $3 \%$ \\
\hline Hong Kong, China & $2 \%$ \\
\hline India & $2 \%$ \\
\hline Emiratos Árabes Unidos & $2 \%$ \\
\hline Australia & $2 \%$ \\
\hline Arabia Saudita & $2 \%$ \\
\hline
\end{tabular}

\begin{tabular}{|l|c|}
\hline \multicolumn{1}{|c|}{ Pais importador } & $\begin{array}{c}\text { Participación en exportaciones } \\
\text { totales de ASEAN (2015) }\end{array}$ \\
\hline China & $13 \%$ \\
\hline Estados Unidos & $12 \%$ \\
\hline Japón & $9 \%$ \\
\hline Hong Kong, China & $6 \%$ \\
\hline Malasia & $5 \%$ \\
\hline Singapur & $5 \%$ \\
\hline República de Corea & $4 \%$ \\
\hline Indonesia & $4 \%$ \\
\hline Tailandia & $4 \%$ \\
\hline India & $4 \%$ \\
\hline Australia & $3 \%$ \\
\hline Taipei Chino & $3 \%$ \\
\hline Alemania & $3 \%$ \\
\hline Viet Nam & $2 \%$ \\
\hline Países Bajos & $2 \%$ \\
\hline
\end{tabular}

Fuente: elaboración propia a base de Trade Map.

En lo referente a la Inversión Extranjera Directa (IED) recibida, si bien una gran parte proviene de los miembros del propio bloque y de la Unión Europea, el $34 \%$ del total la IED entrante proveniente de los socios ASEAN +1. Entre estos, Ja- 
ReVista PUCE, ISSN 1390-7719. Núm.103. 3 de mayo de 2016-

3 De noviembre de 2016. Natalia De María,

María Eugenia Pereira.PP. 273-297

pón que representa el 15\%, seguido por China con una participación de $7 \%$.

CUADRO 8

IED RECIBIDA POR ASEAN EN 2015

\begin{tabular}{|l|c|c|}
\hline \multicolumn{1}{|c|}{ Pais } & $\begin{array}{c}\text { Millones de US\$ } \\
\mathbf{( 2 0 1 5 )}\end{array}$ & Participación \\
\hline ASEAN & 21.938 & $18 \%$ \\
Unión Europea & 19.640 & $16 \%$ \\
Japón & 17.324 & $15 \%$ \\
Estados Unidos & 12.185 & $10 \%$ \\
China & 8.161 & $7 \%$ \\
República de Corea & 5.669 & $5 \%$ \\
Australia & 5.177 & $4 \%$ \\
Nueva Zelanda & 2.241 & $2 \%$ \\
India & 1.252 & $1 \%$ \\
\hline Total IED recibida por ASEAN & 119.412 & $100 \%$ \\
\hline
\end{tabular}

Fuente: elaboración propia a base de ASEAN Statistics.

El aumento de la IED hacia ASEAN en los últimos años, se relaciona directamente con su creciente participación en las cadenas globales de valor de Asia Pacífico lideradas por China. La inserción del bloque a estas cadenas conjuntamente con una estrategia de cooperación conjunta promovió la participación de los miembros en estas, lo cual contribuye al crecimiento de la IED y acompañan al crecimiento económico y desarrollo productivo de la región. Su progresiva apertura comercial promovió la inserción en las cadenas regionales y globales de valor, a partir de la liberalización tanto en bienes como en servicios e inversiones, lo cual profundizó la relación comercial con sus principales socios regionales, los mismos que aumentaron su inversión en los países de la ASEAN.
Dado que el intercambio comercial, así como el flujo de inversiones es mayor con China, Corea y Japón, es que se crea el foro ASEAN +3, que reúne a los miembros de la ASEAN junto a estos tres países en el ámbito de las cumbres de Jefes de Estado y de Gobierno. Para la profundización de su relación plasmaron un lineamiento estratégico. Asimismo, la ASEAN junto con sus seis socios ASEAN + 1 crearon otra instancia de diálogo denominada ASEAN+6 donde se reúnen para promover el intercambio y cooperación entre ellos. Estas reuniones fueron la base para la negociación de un mega-acuerdo entre estas 16 economías asiáticas, el Regional Comprehensive Economic Partnership (RCEP), que lideró China. Este acuerdo apunta a conformar una zona de libre comercio y profundizar el compromiso entre ellos en diversas áreas y es visto como una alternativa al TPP que lidera por Estados Unidos (Urmeneta, 2013). En cuanto al RCEP, se espera que el acuerdo pueda alcanzarse en el correr del año 2016. Se debe tener en cuenta que miembros del futuro RCEP también forman parte del TPP (Australia, Brunéi, Japón, Malasia, Vietnam, Singapur y Nueva Zelanda), cuatro de ellos miembros de la ASEAN.

Pero el bloque no solo se relaciona con sus socios de Asia Pacífico, sino que también estrecha el vínculo con otros importantes socios comerciales. La 
profundización del diálogo e intercambio con la Unión Europea es significativa. En sus comienzos participaban de las reuniones 15 países europeos y los siete miembros que tenía en ese año la ASEAN, junto con China, Corea, Japón y la Comisión Europea. En la actualidad participan de las reuniones 53 Jefes de Estado y Gobierno, dentro de los que se encuentran países como China, Estados Unidos, Rusia, Australia, Nueva Zelanda, Japón y Pakistán, entre otros, lo que demuestra el éxito del diálogo interregional. Las negociaciones para alcanzar una zona de libre comercio entre los bloques comenzaron en el año 2007 pero dos años más tarde fueron pausadas de mutuo acuerdo; a partir de 2013, el Comisionado de Comercio de la Unión Europea indicó que se volverían a retomar las negociaciones en forma individual con cada uno de los miembros de la ASEAN y se consideraría la posibilidad de retomar las negociaciones para una zona de libre comercio entre la ASEAN y la Unión Europea una vez alcanzada la Comunidad (ASEAN, 2015b).

El diálogo con Estados Unidos progresó desde que comenzó en el año 1977. En relación con la cooperación económica entre ambos, en el año 2006 firmaron un acuerdo de comercio e inversiones, Trade and Investment Framework Arrangement (TIFA) y se creó un Consejo Conjunto para implementar el acuerdo. En 2012, se firma otra iniciativa Expanded Economic Engagement (E3) con miras a profundizar la relación económica comercial y crear nuevas oportunidades. En el acuerdo también se apunta a profundizar la cooperación sobre facilitación de comercio, PYMES y armonización de normas entre otros. (ASEAN, 2016a). La relación comercial entre Estados Unidos y la ASEAN tiene un importante componente que son las cadenas globales de valor; Estados Unidos como fuente de demanda y la ASEAN de producción.

Otros socios importantes son Rusia (ASEAN, 2016b), con quien en 2005 se firma un acuerdo sobre cooperación y desarrollo mutuo en temas económicos, comerciales, de seguridad y sociales; Pakistán (ASEAN, 2013) con quien sostiene un diálogo sectorial desde 1993 y Canadá (ASEAN, 2016c), país con el que la cooperación data de 1977 y que en 2011 se aprueba una declaración conjunta sobre comercio e inversiones, así como un plan de trabajo guía para las relaciones entre ambas partes para el período 2016 $-2020$.

Asimismo, con América Latina, el intercambio y cooperación es aún muy bajo (Urmeneta, 2013); sin embargo, la Alianza del Pacífico (Chile, Colombia, Perú y México) ya tuvo reuniones con la ASEAN y tiene un claro interés en la región de Asia Pacífico. El intercambio comercial con el MERCOSUR es también 
ReVista PUCE, ISSN 1390-7719. Núm.103. 3 de mayo de 2016-

3 De noviembre de 2016. Natalia De María, María Eugenia Pereira.PP. 273-297

aún muy pobre y su relación incipiente; si bien se realizó una Reunión Ministerial en 2008 entre ambos bloques, no se dan mayores avances.

La interacción de la ASEAN con otros países más allá de la profundización de las relaciones económicas, comerciales y políticas entre sus miembros, deja ver la importancia que tiene para el bloque la inserción internacional y el posicionarse como un referente a nivel global. Asimismo, el bloque apunta a cerrar acuerdos con sus principales socios co- merciales, para beneficiar así el intercambio entre ellos y que facilite su inserción en las cadenas globales de valor.

\section{Estrategias de inserción de los bloques}

Ambos bloques cuentan con desempeños diferentes en cuanto a su posicionamiento en el escenario comercial internacional. En el siguiente cuadro se puede apreciar alguna de estas diferencias.

\section{CUADRO 9}

COMPARACIÓN ENTRE EL MERCOSUR Y LA ASEAN

\begin{tabular}{|c|c|c|}
\cline { 2 - 4 } \multicolumn{1}{c|}{} & MERCOSUR & ASEAN \\
\hline Año de creación & 1991 & 1967 \\
\hline alcanzado & $\begin{array}{c}\text { En su tratado originario plantean } \\
\text { alcanzar un mercado común. Sin } \\
\text { embargo, actualmente podría ser } \\
\text { considerado como una unión } \\
\text { aduanera imperfecta. }\end{array}$ & $\begin{array}{c}\text { Zona de Libre Comercio vigente } \\
\text { desde 1992. Mercado Común en } \\
\text { proceso de implementación desde } \\
2015 .\end{array}$ \\
\hline $\begin{array}{c}\text { Cantidad de acuerdos } \\
\text { firmados }\end{array}$ & 11 & 5 \\
\hline firses con los que ha & $\begin{array}{c}\text { Chile, Bolivia, México, Perú, } \\
\text { Colombia, Ecuador, India, Israel, } \\
\text { Egipto, Palestina y SACU. }\end{array}$ & $\begin{array}{c}\text { Australia y Nueva Zelanda, China, } \\
\text { Corea, India, Japón. }\end{array}$ \\
\hline Importancia de los & $\begin{array}{c}\text { Los acuerdos firmados no representan } \\
\text { un porcentaje importante del } \\
\text { comercio global del bloque. }\end{array}$ & $\begin{array}{c}\text { Los países con los que se tiene } \\
\text { acuerdo son los principales socios } \\
\text { comerciales del bloque. }\end{array}$ \\
\hline Flexibilizaciones en la \\
negociaciones & $\begin{array}{c}\text { Los países deben negociar en bloque } \\
\text { según la decisión 32/00. Sin embargo, } \\
\text { se ha permitido a Uruguay firmar un } \\
\text { acuerdo con México a modo de } \\
\text { excepción. }\end{array}$ & $\begin{array}{c}\text { Las negociaciones se realizan en } \\
\text { diferentes velocidades y atendiendo } \\
\text { a las necesidades e intereses de } \\
\text { cada uno de los miembros. }\end{array}$ \\
\hline Unión Europea e India. & $\begin{array}{c}\text { Estados Unidos y RCEP. Con la UE se } \\
\text { relanzarán las negociaciones una vez } \\
\text { alcanzada la Comunidad. }\end{array}$ \\
\hline
\end{tabular}

Fuente: Elaboración propia.

Para realizar el análisis, se compararon los bloques desde las siguientes variables:
- Instrumento de integración que se adoptó

- Acuerdos firmados con terceros 
- Flexibilización en las negociaciones

\subsection{Instrumentos de integración adoptados por los bloques}

La ASEAN surge en 1967 con un enfoque en el cual la estabilidad y la paz eran la prioridad principal que movían a los miembros a integrarse. El bloque asiático está compuesto por diez países que tienen realidades económicas, sociales, culturales, religiosas y políticas muy diferentes; $\sin$ embargo, esto no fue excusa para avanzar hacia la profundización de la integración. Si bien la creación de la ASEAN data de varias décadas antes del surgimiento del MERCOSUR, el proceso del sudeste de Asia convierte el tema económico comercial en uno de sus pilares con la creación de la zona de libre comercio denominada Asean Free Trade Agreement (AFTA) en el año 1992, un año después de la firma del Tratado de Asunción. El contexto internacional en el cual el AFTA surge se comparte con el MERCOSUR, ya que esta data de 1991; sin embargo, la evolución de los mismos es diferente.

El instrumento de integración que eligió cada uno va a tener implicancias en la estrategia de inserción que adoptó cada bloque. A pesar que ambos tienen como objetivo final la concreción de un mercado común, plantean una hoja de ruta diferente para alcanzarlo. En primer término, el MERCOSUR propone un recorrido ya probado por otros procesos de integración, que consiste en conformar en primera instancia una zona de libre comercio seguida de una unión aduanera y una vez completadas esas etapas, dar el salto hacia el mercado común. Luego las decisiones que se tomaron en este sentido tienen como base lo que se planteó en el Tratado de Asunción, en donde se establecía el objetivo de alcanzar la libre movilidad de factores y la conformación de un arancel externo común, además de otros instrumentos típicos de una unión aduanera.

En cambio, la ASEAN optó en una primera instancia por formar una zona de libre comercio. Como se mencionó anteriormente, es recién con la declaración de la Visión 2020 que el bloque decide avanzar hacia el mercado común, pero sin la necesidad de conformar previamente una unión aduanera. Este va a ser un factor crucial para la concreción de acuerdos en un proceso donde los diez miembros cuentan con asimetrías estructurales y políticas marcadas y difieren en los intereses a la hora de negociar.

\subsection{Acuerdos firmados con terceros}

En cuanto a la cantidad de acuerdos, se puede observar que el MERCOSUR cerró una mayor cantidad de tratados con respecto a la ASEAN. En este sentido, ambos bloques se centraron en acercarse primero a su región. La dife- 
Revista PUCE, ISSN 1390-7719. Núm.103. 3 de MAYO de 2016 -

3 De noviembre de 2016. Natalia De María,

María Eugenia Pereira.PP. 273-297

rencia radica en el peso que tienen estos mercados para el comercio total del bloque, en donde los acuerdos que se alcanzaron por el bloque asiático, tienen mayor importancia. Es decir, la ASEAN cerró menos acuerdos, pero lo hizo con sus principales socios comerciales. Por su parte, el MERCOSUR lo hizo con la región, y estos acuerdos tienen un peso relativamente importante. Asimismo, concretó tratados con otros países, pero estos se pueden catalogar más como gestos políticos por su bajo interés comercial.

Si se toman en cuenta las negociaciones en curso, en el caso del MERCOSUR, se deben mencionar la que se cuenta con la Unión Europea para la concreción de un tratado de libre comercio y la inminente profundización del acuerdo con India. Sobre estos, se puede decir que representan buenas noticias para el bloque. La importancia geopolítica de India lo vuelve un actor clave en el escenario internacional y sus características lo convierten en un mercado importante para el comercio internacional. Ampliar el acuerdo que se tiene, se vuelve un factor crucial.

En cuanto a las negociaciones de la ASEAN, el bloque asiático no se queda afuera de la tendencia internacional de las meganegociaciones. De hecho, Brunéi, Singapur, Malasia y Vietnam son miembros del TPP, acuerdo recientemente firmado y que los acerca a Estados Unidos, líder del bloque. Además, la ASEAN más todos los países que cuentan con un acuerdo ASEAN +1 impulsaron el RCEP, estrategia de China para no perder el liderazgo en la región y de cierta forma, contraponerse al TPP.

Asimismo, con la Unión Europea las negociaciones comenzaron en el 2007, pero fueron suspendidas el año 2009 y serán retomadas las negociaciones como bloque una vez que se alcance la Comunidad. Sin embargo, en el 2013, la UE propuso negociar en un formato bilateral con cada uno de los miembros. Nuevamente, el pragmatismo para negociar que se suma a la facilidad de no contar con una unión aduanera entre los países, hizo que el bloque asiático logre tener negociaciones activas con las principales economías. Así es como, la iniciativa E3 permite al bloque tener diálogo directo con Estados Unidos en donde se busca la expansión de las inversiones y el comercio entre las partes.

\subsection{Flexibilización en las negociacio- nes}

En cuanto a la flexibilización a la hora de negociar, el MERCOSUR plantea desde un inicio la importancia de la negociación en conjunto, lo que sin lugar a duda aumenta el poder negociador de los socios más pequeños del bloque, a pesar que se le otorgó a Uruguay una excepción para cerrar un acuerdo con México, país que también pertenece a la ALADI. A pesar de eso, el bloque reafirmó su posición ante las negociaciones 
en conjunto en varias oportunidades y un ejemplo de ello es la Decisión $N^{\circ}$ 32/00, así como la negativa que brinda el bloque ante el potencial acercamiento de forma bilateral de algunos de los países miembros con un tercero (fue el caso de Estados Unidos con Uruguay). Este aspecto se cuestiona duramente por algunos de los Estados parte, así como por sectores de la industria que consideran que su país tendría mejor oportunidad si se concretasen más tratados de libre comercio, como es la posición de la Federação das Indústrias do Estado de São Paulo (FIESP), que alienta a que su país se involucre en más negociaciones internacionales en pos de aumentar los mercados a los que podría acceder con un costo menor.

Estos reclamos se apoyan en un cambio de contexto regional, en el cual se debe considerar los problemas económicos e institucionales de Brasil, que cuenta actualmente con un Presidente interino tras la suspensión de Rousseff. Asimismo, el giro de 180 grados que dio la política exterior argentina con la asunción del Presidente Macri y los continuos reclamos de Uruguay de apostar por un regionalismo abierto, en que los países vecinos ocupen un rol principal en la inserción internacional, pero sin descuidar el resto del mundo. La flexibilización que se quiere imponer al MERCOSUR permitiría contemplar los intereses de los actores en cuanto a la apertura de nuevos mercados, pero atentaría contra el modelo de integración elegido por el bloque desde el Protocolo de Ouro Preto.

El MERCOSUR tiene un ejemplo cercano, la CAN, que habilitó a través de la Decisión Nº 598 la negociación en distintas velocidades con el fin de asegurar la concreción de negociaciones que incluyan los intereses de países con realidades muy diferentes. Actualmente la CAN tiene su unión aduanera suspendida y se encuentra en una posición crítica. Esta situación no es culpa exclusivamente de la mencionada decisión, sino que otros factores contribuyeron a la misma, como por ejemplo el alejamiento de algunos de sus miembros, como Venezuela (miembro pleno del MERCOSUR actualmente) y como Bolivia que se encuentra a la espera de la ratificación de los Estados miembros del MERCOSUR para poder ingresar. Esto hace que el proceso de integración sudamericano piense con cautela antes de tomar una decisión que claramente desestimulará la concreción de parte de sus objetivos originarios. Sin embargo, sin un sinceramiento sobre hasta dónde puede llegar la comunión de los países miembros, el bloque continuará en un estado de estancamiento que ya lleva varios años.

Por su lado, la ASEAN para compensar sus asimetrías, tuvo que adaptar los modelos de negociación con terceros países para que se contemplen las necesidades e intereses de cada uno. De esta 
Revista PUCE, ISSN 1390-7719. Núm.103. 3 de MAYO de 2016 -

3 De noviembre de 2016. Natalia De María,

María Eugenia Pereira.PP. 273-297

forma lograron firmar acuerdos con las principales economías asiáticas como China, Japón, Corea e India, así como también con Australia y Nueva Zelanda. Pero aún más distinguible es que lograron potenciar estos acuerdos para generar encadenamientos productivos y formar parte de las cadenas globales de valor entre los miembros, aspecto que se encuentra aún poco desarrollado en el MERCOSUR.

\section{CONCLUSIÓN}

La estrategia de inserción comercial del MERCOSUR no es del todo clara. No hay una política común que resalte los intereses del bloque por sobre los intereses de los países. Si bien en los diferentes discursos de las altas autoridades de los miembros suelen mencionar la misma, no existe documento que plasme las acciones que se deben seguir para colocar el MERCOSUR en el escenario internacional.

En cuanto a la ASEAN, existe una inserción internacional exitosa. En parte, esto se debe a la posición geográfica en que se encuentra, que coloca al bloque cerca de los principales centros de consumo y que la convierte en un importante centro de producción, pero también gracias a la estrategia pragmática y flexible que llevan adelante.

Por su parte, el MERCOSUR y sus miembros aún no logran reaccionar ante el nuevo contexto del comercio internacional como sí lo hicieron otros países latinoamericanos y continúa aún con un accionar que no arroja resultados beneficiosos. El futuro de ambos bloques dependerá de la capacidad de adaptarse a los cambios que se producen en el escenario internacional en sus diferentes áreas y es justamente la ASEAN la que demostrará una mayor resiliencia para adaptarse a los cambios que se dieron en los últimos tiempos en la integración económica. 


\section{BIBLIOGRAFÍA}

ASEAN (1997) ASEAN Vision 2020. Disponible en: http://www.asean.org/?static_post=asean-vision-2020

ASEAN (2003) Declaration of ASEAN Concord II (Bali CONCORD II). Disponible en: http://www. asean.org/?static_post=declaration-of-asean-concord-ii-bali-concord-ii

ASEAN (2007) ASEAN Charter. Disponible en: http://www.asean.org/storage/images/archive/publications/ ASEAN-Charter.pdf

ASEAN (2013) Overview of ASEAN-Pakistan Sectoral Dialogue Cooperation. Disponible en: http:// asean.org/?static_post=overview-of-asean-pakistan-sectoral-dialogue-cooperation-2

ASEAN (2015a) ASEAN Economic community blueprint 2025. Disponible en: http://www.asean.org/storage/2016/03/AECBP_2025r_FINAL.pdf

ASEAN (2015b) Overview of ASEAN-EU Dialogue Relations. Disponible en: http://asean.org/?static_pos$\mathrm{t}=$ overview-of-asean-eu-dialogue-relations

ASEAN (2016a) Overview of ASEAN - U.S. dialogue relations. Disponible en: http://www.asean.org/wp-content/uploads/2016/01/4Jan/
Overview-of-ASEAN-US-Dialogue-Relations-(4-Jan-2016).pdf ASEAN (2016b) Overview ASEAN - RUSIA dialogue partnership. Disponible en: http://www.asean.org/ wp-content/uploads/2016/01/ 4Jan/Overview-ASEAN-RussiaJanuary-2016-cl.pdf

ASEAN (2016c) Overview of ASEAN-Canada Dialogue Relations. Disponible en: http://asean.org/ storage/2012/05/Overview-ofASEAN-Canada-Dialogue-Relations-17-May-2016-clean.pdf

Bartesaghi, I. (2010a). Cuadragésimo octavo y quincuagésimo quinto protocolo adicional del Acuerdo de Complementación Económica no35 (Uruguay - Chile). Departamento de Integración y Comercio Internacional, Cámara de Industrias del Uruguay. Disponible en http://www.ciu.com.uy/innovaportal/file/429/1/uruguay---chile. pdf

Bartesaghi, I. (2010b). Acuerdo Preferencial entre MERCOSUR y la República de la India. Departamento de Integración y Comercio Internacional, Cámara de Industrias del Uruguay. Disponible en http:// www.ciu.com.uy/innovaportal/ file/429/1/MERCOSUR_-_india. pdf 
Revista PUCE. ISSN 1390-7719. Núm.103. 3 de mayo de 2016 -

3 De noviembre de 2016. Natalia De María,

María Eugenia Pereira.PP. 273-297

Bartesaghi, I. (2015a). La estrategia comercial de Estados Unidos y la Unión Europea en América Latina: impactos para el MERCOSUR. Magro: Montevideo.

Bartesaghi, I. (2015b). Algunas reflexiones sobre el MERCOSUR. Propuestas para una necesaria reformulación. En Análisis y perspectivas, №3, Konrad Adenauer Stiftung: Chile

Bartesaghi, Ignacio (2014a) La Asociación de Naciones del Sudeste Asiático: algunas similitudes con la Unión Europea. Publicado en el libro Los procesos de integración como factor de paz, Universidad Externado de Colombia, primera edición Disponible en: http:// www.ucu.edu.uy/sites/default/ files/pdf/2015/asociacion_naciones_sudeste_asiatico_similitudes_union_europea.pdf

Bartesaghi, Ignacio (2014b) La ASEAN y el MERCOSUR: similitudes, diferencias y potencialidades. Publicado en Revista Temas de Derecho Económico. Enfoque Nacional e Internacional. №2, por Tribunales Ediciones, Buenos Aires. Disponible en: http://www.ucu.edu.uy/ sites/default/files/facultad/fce/ dnii/La_ASEAN_y_el_MERCOSUR_similitudes_diferencias_y_ potencialidades.pdf
Bizzozero, L. Vera, T. (1995). El MERCOSUR en funcionamiento: de Asunción a Ouro Preto. Documento de Trabajo no 1/95. Departamento de Economía. Facultad de Ciencias Sociales, Montevideo.

Khoman, Thanat (1992) ASEAN Conception and Evolution. Disponible en: http://www.asean. org/?static_post=asean-conception-and-evolution-by-thanat-khoman

Peña, F. (1996). La construcción del MERCOSUR. Lecciones de una experiencia. En Archivos del Presente. No 4, Buenos Aires página 113 133.

Urmeneta, Roberto (2013) ASEAN: Interrelaciones y potencialidades con América Latina y el Caribe. Observatorio América Latina Asia Pacífico. Disponible en: http://www.observatorioasiapacifico.com/data/ OBSERVATORIO.Images/Publication/1213/20131101042232ASEAN_Interrelaciones_y_potencialidades_con_ALC.pdf

Vinayak HV, Fraser Thompson, and Oliver Tonby (2014) Understanding ASEAN: Seven things you need to know. McKinsey \& Company. Disponible en: http://www.mckinsey.com/ industries/public-sector/our-insights/understanding-asean-seven-things-you-need-to-know 
